Mon. Not. R. Astron. Soc. 000, 000-000 (0000) Printed 6 March $2017 \quad$ (MN LATEX style file v2.2)

\title{
Change in General Relativistic Precession Rates due to Lidov-Kozai oscillations in Solar System
}

\author{
A. Sekhar ${ }^{1,2 *}$, D. J. Asher ${ }^{2}$, S. C. Werner ${ }^{1}$, J. Vaubaillon ${ }^{3}$, G. $^{4}{ }^{4}$ \\ ${ }^{1}$ Centre for Earth Evolution and Dynamics, Faculty of Mathematics and Natural Sciences, University of Oslo, Blindern N-0315, Norway \\ ${ }^{2}$ Armagh Observatory and Planetarium, College Hill, Armagh BT61 9DG, United Kingdom \\ ${ }^{3}$ IMCCE, Observatoire de Paris, 77 Avenue Denfert Rochereau, F-75014 Paris, France \\ ${ }^{4}$ Harvard-Smithsonian Center for Astrophysics, Cambridge, MA, USA \\ *E-mail: aswin.sekhar@geo.uio.no,asw@arm.ac.uk
}

Accepted: ; Received: ; In Original Form: ; MNRAS

\begin{abstract}
Both General Relativistic (GR) precession and the Lidov-Kozai mechanism, separately, are known to play an important role in the orbital evolution of solar system bodies. Previous works have studied these two mechanisms independently in great detail. However, both these phenomena occurring at the same time in real solar system bodies have rarely been explored. In this work, we find a continuum connecting the GR precession dominant and Lidov-Kozai like mechanism dominant regimes, i.e. an intermediate regime where the competing effects of GR precession and Lidov-Kozai like oscillations co-exist simultaneously. We find some real examples in the solar system in this intermediate regime. Moreover we identify a rare example amongst them, comet 96P/Machholz 1, which shows significant changes in the rates of GR precession (an order of magnitude higher than Mercury's GR precession rate) due to sungrazing and sun colliding phases induced by Lidov-Kozai like oscillations. This comet's combination of orbital elements and initial conditions (at the present epoch) favour this measurable rapid change in GR precession (at some points peaking up to 60 times Mercury's GR precession rate) along with prograde-retrograde inclination flip (due to Lidov-Kozai like oscillations). Similar tests are performed for hundreds of bodies lying in the moderately low perihelion distance and moderately low semi-major axis phase space in the solar system, the present lowest perihelion distance asteroid $322 \mathrm{P} / \mathrm{SOHO}$ 1 , and further examples connected with 96P/Machholz 1 namely, the Marsden and Kracht families of sungrazing comets plus low perihelion meteoroid streams like Daytime Arietids (ARI) and Southern Delta Aquariids (SDA).
\end{abstract}

Key words: General relativistic precession, Lidov-Kozai oscillations, comets, asteroids, meteoroids, satellites

\section{INTRODUCTION}

Two well known phenomena associated with low perihelion distance bodies in orbital dynamics are general relativistic (GR) precession and Lidov-Kozai oscillations.

The accurate prediction of the perihelion shift of Mercury in accord with real observations is one of the significant triumphs of the general theory of relativity developed by Einstein (1915). Past works have looked into the GR precession in perihelion in different types of solar system bodies like planets (Weinberg 1972; Brumberg 1991; Quinn, Tremaine \& Duncan 1991; Iorio 2005), asteroids (Sitarski 1992), comets (Shahed-Saless \& Yeomans 1994) and meteoroid streams (Fox, Williams \& Hughes 1982; Sekhar 2013; Galushina, Ryabova \& Skripnichenko 2015). More recently some works have explored the cases of GR precession in exoplanetary systems (Naoz et al. 2011, 2013; Li et al. 2015).

A dynamical mechanism first found by Lidov (1962), and further studied by Kozai (1962) who applied it to sunJupiter-asteroid 3-body systems, explains the periodic exchange between eccentricities $e$ and inclinations $i$ thereby increasing or decreasing the perihelion distance $q$ secularly in the orbiting body. In its purest form the Lidov-Kozai mechanism involves three bodies, namely a central body, test particle and perturber. In real situations such as the solar system, where the perturber may be interior or exterior (page 154, Morbidelli 2011) to the test particle and/or there may be higher order multiplicity effects (due to perturbations from other giant planets than Jupiter, lesser in strength 
compared to Jovian perturbations), pure Lidov-Kozai still offers a convenient way to understand the dynamical behaviour. We shall use the term Lidov-Kozai like mechanism to cover these situations.

This mechanism has been related to the rapid change in orbits of artificial satellites (Lidov 1962) around the Earth. Past works have shown that the Lidov-Kozai like mechanism can lead to a flip in orbits i.e. inclinations switching from prograde to retrograde or vice-versa (Naoz et al. 2011; Lithwick \& Naoz 2011; Naoz et al. 2013; Li et al. 2014b) during the body's secular evolution. Naoz et al. (2011) discussed the possibility of orbital flips in hierarchical triple body systems for the first time in the context of exoplanet systems. Bailey, Chambers \& Hahn (1992) found that Lidov-Kozai (here it is not pure Lidov-Kozai mechanism in strict terms but higher order multiplicity effects) is an efficient way by which asteroidal and cometary orbits could land up in sunskirting or sungrazing orbits. The mechanism is known to have an important role in the long term evolution of different classes of small bodies (Vaubaillon, Lamy \& Jorda 2006; Granvik, Vaubaillon \& Jedicke 2012) in the context of impact studies (Werner \& Ivanov 2015) due to the Lidov-Kozai like cycles in orbital elements which lead to complications in using analytical and numerical techniques (Manley, Migliorini \& Bailey 1998) to compute impact probabilities from small bodies on planets. More recently there are examples found in the exoplanetary systems (Naoz et al. 2013; Li et al. 2014a; Naoz 2016) which undergo Lidov-Kozai like oscillations around the central body thus showing the generality of this phenomenon in any suitable dynamical system.

Of the past studies mentioned above, the ones that pertain to the solar system have generally related to either of the two phenomena separately, not GR and Lidov-Kozai like effects happening at the same time. Moreover previous works in exoplanet systems have indicated that GR precession can suppress Kozai like oscillations in some cases ( $\mathrm{NaOz}$ et al. 2013) depending on the orbital elements phase space. Hence it has been shown that one phenomenon can compete or dominate over another in exoplanet systems. Again, this particular idea has not been well explored in the context of real solar system bodies. Naoz et al. (2013) showed that inclusion of GR precession can lead to re-triggering eccentricity excitations and orbital flips thereby pointing to the situation of both GR precession and Kozai effect co-existing at the same time in exoplanet systems.

In this work, we find real examples of solar system bodies where significant (to be quantified later) GR precession and Lidov-Kozai like behaviour can co-exist and complement each other, forming a continuum between the two regimes where the respective separate effects dominate. The LidovKozai like mechanism leads to secular lowering of $q$ which in turn leads to a huge increase in GR precession of argument of pericentre $\omega$. This in turn gives feedback to the LidovKozai like mechanism as the $e, i$ and $\omega$ cycles are closely correlated.

A real solar system body, 96P/Machholz 1, exhibiting these trends by combining the two dynamical effects during present times is identified out of the hundreds of small bodies lying in the moderately low $q$ and moderately low $a$ (semimajor axis) phase space. This particular effect would be more pronounced especially when a body gradually evolves into a sungrazing and sun colliding orbit. The dynamical evolution becomes even more interesting when inclination flips occur, the secular Lidov-Kozai like reduction in $q$ applying in both prograde and retrograde cases.

For all calculations in this paper, Newtonian n-body integrations were done using Chambers' (1999) MERCURY package incorporating the RADAU (Everhart 1985) and MVS (Mixed-Variable Symplectic: Wisdom, Holman \& Touma 1996) algorithms. For GR cases, MERCURY integrations included an additional sub-routine (provided by $\mathrm{G}$. $\mathrm{Li}$ ) incorporating the GR corrections (Benitez \& Gallardo 2008) in the n-body code; the MVS algorithm was used. The RADAU accuracy parameter was set to $10^{-16}$ and the MVS time step was chosen as one day. Each asteroid, comet and meteoroid particle was treated as a test particle (i.e. zero mass) and integrated in the combined presence of the sun and eight planets Mercury to Neptune. Non-gravitational forces were not considered in comets, nor radiative forces for asteroids and meteoroid streams, so that we can distinguish GR active and Kozai like dynamics active spaces without additional complicating effects. In the GR-included integrations, GR effects were taken into account for the evolution of all the big bodies (i.e. planets) and small bodies involved. In the Newtonian integrations, GR did not act on either big or small bodies. For all integrations the initial orbital elements (taken from JPL-Horizons: Giorgini et al. 1996) and initial epoch (JD 2451000.5) of the eight planets remained the same, for uniformity in comparisons. Hence every GR case presented here, and likewise every Newtonian case, is based on an identical solar system model. Cross-checks between RADAU and MVS Newtonian integrations confirmed that either integrator could reproduce the same orbital evolution. Searches for small bodies with various ranges of orbital elements used IAU-MPC (Minor Planet Center) and initial elements of specific bodies to be integrated were the latest epoch available from JPL-Horizons.

\section{LIDOV-KOZAI LIKE MECHANISM DOMINANT CASES}

Long term Lidov-Kozai like oscillations can be found in different examples of sungrazing comets (Bailey, Chambers \& Hahn 1992), bringing these comets closer and closer to the sun after every perihelion passage and $q$ being lowered secularly. Although the perihelion distances stay in the range of GR-active space and can, in principle, increase the GR precession rates (due to decrease in $q$ ), the orbital periods $P$ (and $a$ ) mostly stay way beyond the significant GR-active space. For GR effects happening near the perihelion passage trajectory to accumulate efficiently over time, the body needs a relatively small orbital period so that perihelion passages happen frequently. Hence for most known bodies this GR precession increase is insignificant and difficult to separate and confirm from observations. In this section, we talk about single orbit timescales of the order of $\sim 10^{2}-10^{6} \mathrm{yr}$, so that even if GR precession per orbit is significant, the accumulated effect over these timeframes is low.

In this work we quantify GR precession as significant if it is comparable to that of Earth. For Earth's present orbit $\Delta \omega \sim 3.8$ arc seconds per century or $\Delta \omega \sim 0.038$ arc seconds per perihelion passage (table 8.3, page 198, Weinberg 1972) which has been verified and confirmed by observations. 
Table 1. Initial conditions for the bodies presented as plots in this work. All taken from JPL-Horizons except for meteoroid stream Daytime Arietids (ARI) from IAU-Meteor Data Center.

$\begin{array}{ccccccccc} & \text { Epoch JDT } & a & e & i & \omega & \Omega & M & \text { Fig. } \\ \text { C/1932 G1 } & 2426760.5 & 45.00523804 & 0.972127 & 74.277600 & 303.5157 & 213.4835 & 359.980648 & 1 \\ \text { 2011 CL50 } & 2455606.5 & 0.88303406 & 0.147614 & 0.173164 & 285.543879 & 22.791201 & 201.817484 & {[2} \\ \text { 96P } & 2456541.5 & 3.03393972 & 0.959211 & 58.312214 & 14.757748 & 94.323236 & 77.992760 & {[3} \\ \text { ARI } & 2457546.5 & 2.67 & 0.974 & 27.7 & 28.7 & 79.1 & 0.0 & {[4} \\ \text { 322P } & 2457179.5 & 2.51626146 & 0.978676 & 12.589235 & 49.049476 & 359.524487 & 337.760658 & 5 \\ \text { 2008 KP } & 2455509.5 & 1.10063074 & 0.789847 & 59.835824 & 344.973090 & 62.464197 & 293.337766 & {[6} \\ \text { Mercury } & 2451000.5 & 0.38709895 & 0.205620 & 7.005045 & 29.121849 & 48.332314 & 106.519879 & 12\end{array}$

(a) Evolution of Orbital Element
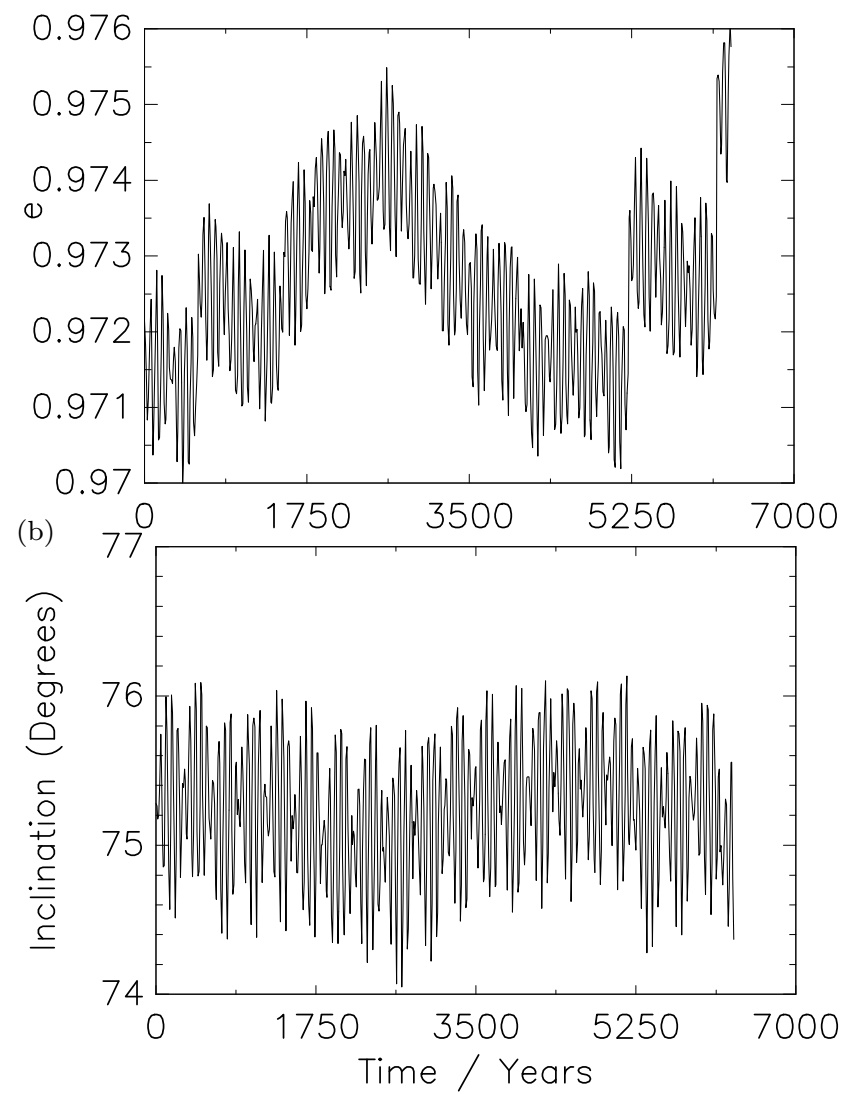

Figure 1. Orbital evolution of (a) eccentricity (b) inclination of C/1932 G1 (Houghton-Ensor) forward for 7 kyr from present. This body gets ejected out of solar system in about $6.3 \mathrm{kyr}$ in our simulations.

GR precession in $\omega$ can be computed using this closed form expression (page 197, Weinberg 1972):

$\Delta \omega=6 \pi G M / a\left(1-e^{2}\right)=6 \pi G M / q(1+e)$

in radians/revolution. It should be noted that the direction of the precession of pericentre is always in the same direction of the motion of the orbiting body (page 197, Weinberg 1972).

In the case of Halley-type comets (with relatively low $q \leq$ perihelion distance of Mercury), the periods (order of $\sim 10^{2}$ yr) are high enough that it is practically difficult to distinguish GR precession effects (from other effects or changes in orbits) after each perihelion passage and moreover, the number of perihelion passages to accumulate GR
Table 2. GR precession in argument of pericentre $\Delta \omega$ per perihelion passage for examples of highly eccentric sungrazing comets (orbital elements taken from sungrazing comets section of Marsden \& Williams 2008). For comparison, the value for Mercury is 0.103 arc seconds/revolution.

\begin{tabular}{lllll}
\hline Body & $\begin{array}{l}q \\
(\mathrm{AU})\end{array}$ & $e$ & $\begin{array}{l}\Delta \omega \\
(\text { arc seconds } \\
\text { per } q \text { passage })\end{array}$ & $\begin{array}{l}P \\
(\mathrm{yr})\end{array}$ \\
\hline \hline C/1979 Q1 & 0.005 & 1 & 4.0 & NA \\
C/1965 S1-A & 0.008 & 1 & 2.5 & 900 \\
C/2008 E7 & 0.055 & 1 & 0.35 & NA \\
C/1997 H2 & 0.136 & 1 & 0.14 & NA \\
\hline
\end{tabular}

precession (during every near $q$ passage) is small per unit of time. However Kozai like oscillations can be easily distinguished in these bodies during their long term evolution and the Kozai like mechanism stays dominant (over GR precession) in these bodies. This point about insignificant GR precession accumulation applies to other minor bodies such as Edgeworth-Kuiper Objects which have relatively high $P \gtrsim 10^{2}$ yr.

Figure 1 shows the $e$ and $i$ evolution of C/1932 G1 (a representative Halley-type low- $q$ comet). Kozai like oscillations can be seen in plots (a) and (b). Tests were repeated for Halley-type comets taken from the cometary catalogue compiled by Marsden \& Williams (2008) and they show insignificant GR precession as expected, Kozai like mechanism dominant over GR precession being the typical behaviour because of the higher $P$.

Long period sungrazers like the Kreutz family of comets (Kreutz 1888) and Meyer group come incredibly close to the sun. In these cases, although the GR perihelion precession per single perihelion passage is at the highest (see Table 2), $P$ is of the range $\sim 10^{3}-10^{6}$ yr (mostly members of the Kreutz family) and hence GR precession accumulating over time is out of the question for the timeframes we discuss in this work. Moreover because of the perturbations from galactic tides and passing stars when these bodies reach near aphelion, it would be difficult to imagine any of these bodies showing a consistent or periodic pattern of GR precession accumulation in comparable magnitude over long time scales.

The examples presented in Table 2 are representatives showing low, intermediate and high $q$ amongst the sungrazing candidates $(e \sim 1)$ in the Marsden \& Williams (2008) comet catalogue. Table 2 shows that lowest $q$ candidate can have GR precession per revolution as much as 40 times that 
Table 3. GR precession in argument of pericentre $\Delta \omega$ per century for some examples of low $q$, low $a$ and low $i$ bodies in solar system. For comparison, the value for Mercury is 43 arc seconds/century.

\begin{tabular}{lllll}
\hline Body & $\begin{array}{l}q \\
(\mathrm{AU})\end{array}$ & $\begin{array}{l}\Delta \omega \\
(\mathrm{AU})\end{array}$ & $\begin{array}{l}\Delta \omega \\
\text { (arcseconds } \\
\text { per century) } \\
\text { (analytic) }\end{array}$ & $\begin{array}{l}\text { (arcseconds } \\
\text { per century) } \\
\text { (integrations) }\end{array}$ \\
\hline \hline 2015 KE & 0.85 & 0.97 & 4.2 & 4.6 \\
2013 BS45 & 0.91 & 0.99 & 4.0 & 4.5 \\
2010 VQ & 0.69 & 0.86 & 5.8 & 6.2 \\
2011 CL50 & 0.76 & 0.89 & 5.3 & 5.9 \\
\hline
\end{tabular}

of Mercury's GR precession per revolution but because of large $P$ of typical sungrazing comets, this precession accumulating efficiently over time is unrealistic for future journeys into the inner solar system. Moreover some extreme sungrazers do not survive for another perihelion passage due to their disruption near the sun or collision with the sun.

However in some particular cases in the solar system, the GR precession can be significant and measurable, while Lidov-Kozai like cycles still survive during the same time (Section 4).

\section{GR PRECESSION DOMINANT CASES}

GR precession in pericentre was first studied for the classic example of Mercury's orbit. More recently there are works (Kerr 1992; Boué, Laskar \& Farago 2012; Lithwick \& Wu 2014) which looked into the long term dynamical behaviour of Mercury and its stability within the solar system. These latest works have indicated that GR precession along with secular resonances are the dominating effects in Mercury's long term evolution ruling out an important role from any Kozai like mechanism. In this section, we talk about GR accumulation with single orbit timescales of the order of $\leq$ $1 \mathrm{yr}$. In these cases, the GR effect is accumulated slowly and steadily over time.

Here we look at the evolution of bodies within $e \leq 0.2$ ( $\sim$ eccentricity of Mercury), $i \leq 7.0$ ( $\sim$ inclination of Mercury) and $a \leq 1.0$ au ( semi-major axis of Earth). This range enlists 115 objects in the IAU-MPC database. Numerical integrations of these bodies show measurable GR precession (which can be tested using comparison of analytical calculations and numerical integrations; see Table 3) and Lidov-Kozai like oscillations at some point in the future if the evolution is followed for a few $10^{5} \mathrm{yr}$. However all the subset of these bodies with $i \leq 3^{\circ}$ (61 objects) do not show (see the example in Figure 2) consistent and long term Lidov-Kozai like oscillations for the near future $\left(\sim 10^{3} \mathrm{yr}\right)$. The bodies shown in Table 3 (the last of which is plotted in Figure 2) are representative of these 61 objects. Because both $e$ and $i$ are low at the same time in these cases compared to bodies discussed in Section 2 the Lidov-Kozai like oscillations do not dominate over other effects like GR precession or secular resonances. It should be noted that orbital timescales depend only on semi-major axis and eccentricity here. But inclination is mentioned and discussed because anti-correlation of $e$ and $i$ is a crucial signature of Kozai like (a) Evolution of Orbital Element

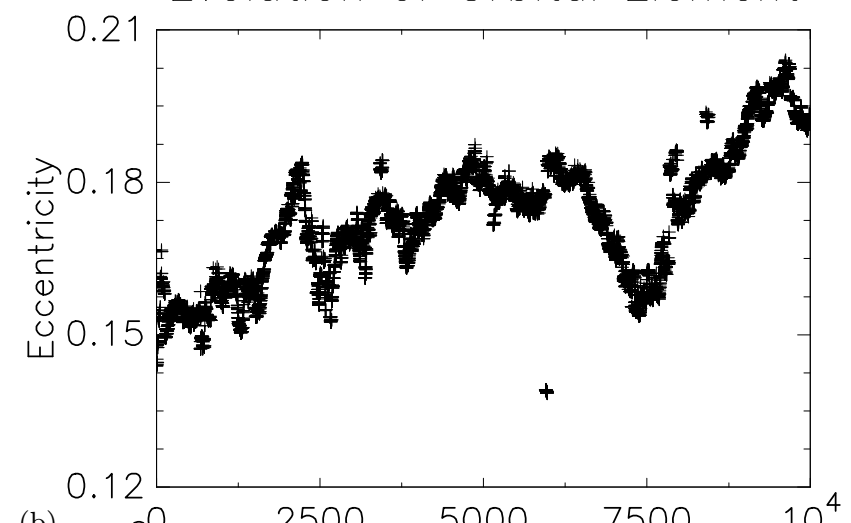

(b)

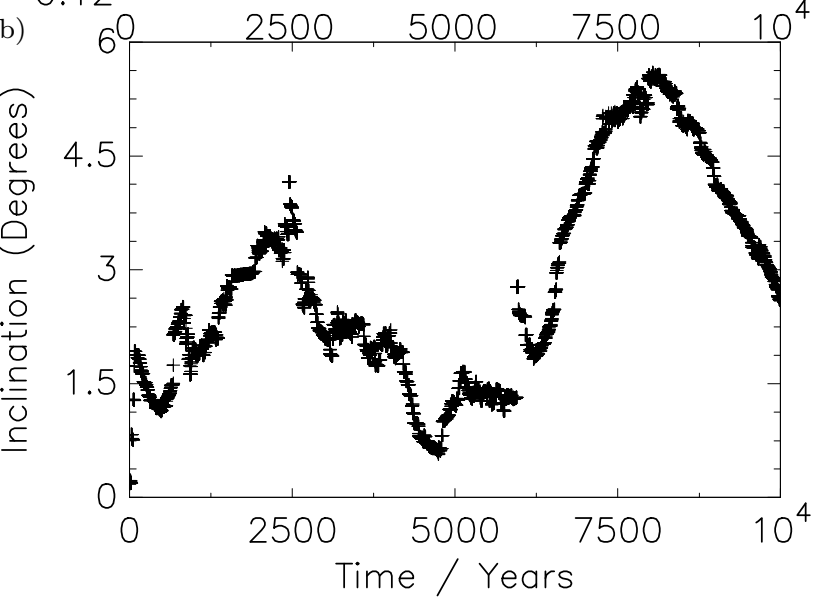

Figure 2. Evolution of $e$ and $i$ of 2011 CL50 for 10 kyr into future.

oscillation and the nature of inclination evolution helps to identify Kozai like behaviour in the solar system.

The $e$ and $i$ evolution of 2011 CL50 (Figure 2) shows no consistent Kozai like oscillations whereas the GR precession is significant (Table 3). This trend is typical for other low $q$, low $a$ and low $i$ bodies from the 61 bodies enlisted and discussed in this section. The cases showing absence of consistent Lidov-Kozai like oscillations (or negative results) are presented here to highlight the fact that there are areas in the solar system where significant GR precession dominates over Kozai like mechanism (similar to the exoplanetary cases presented in $\mathrm{NaOz}$ et al. 2013), in contrast to examples in Section 2 The overlap of these phenomena (GR precession and Kozai like oscillations), complementing each other thereby leading to unique dynamical behaviour, is discussed in detail in Section 4

\section{CO-EXISTENCE OF GR PRECESSION AND LIDOV-KOZAI OSCILLATIONS}

In this section, we consider Lidov-Kozai like cycle timescales of the order of some kyr which contributes to GR enhancement due to sungrazing phases induced by a Kozai like mechanism. We consider bodies with $q \leq 0.3$ au ( perihelion distance of Mercury) and $a \leq 4$ au ( 10 times the semimajor axis of Mercury), thus single orbit timescales of the order of $\leq 8$ years. This condition enlists 244 objects from 
the IAU-MPC database. The same condition was applied to the list of established meteor showers from the IAU-MDC (Meteor Data Center) database. Some representative bodies with relatively high GR precession rates from these lists and their GR precession calculated using analytical formulae and numerical integrations are shown in Table 4.

We were interested to identify bodies evolving in the near future ( $\sim$ thousands of years) into rapid sungrazing and sun colliding phases and undergoing inclination flips, due to Lidov-Kozai like oscillations and being GR active at the same time. Of all the bodies we checked from the IAUMPC, and Marsden plus Kracht families from the comet catalogue (Marsden \& Williams 2008), 96P/Machholz 1 stands out because it shows all these trends in the near future. The uniqueness of $96 \mathrm{P}$ has been reported before in different contexts, its dynamical behaviour having octuple crossing possibilities (Babadzhanov \& Obrubov 1987, 1992a, 1992b, 1992c, similar to the case of another periodic comet Machholz, 141P, discussed in Asher \& Steel 1996). The linkage of the orbit of $96 \mathrm{P}$ with orbits of Extreme Trans Neptunian Objects (ETNOs) has been explored by de la Fuente Marcos, de la Fuente Marcos \& Aarseth (2015). 96P has been linked with two families of sungrazing comets and two meteoroid streams. Because of its previously established connection (Ohtsuka, Nakano \& Yoshikawa 2003, Sekanina \& Chodas 2005) with the Marsden and Kracht sungrazers and low $q$ meteoroid streams like Daytime Arietids (ARI) and Southern Delta Aquariids (SDA), our tests were repeated on all these related objects as well.

Figures 3 a, 3b and 3r, 3d show the near future $e$ and $i$ evolution of $96 \mathrm{P}$ for GR-included and Newtonian-only cases respectively. The test particle undergoes Kozai like oscillation (cf. Abedin et al. 2017) and near the final phase of about $120 \mathrm{yr}$, inclination flip occurs from prograde to retrograde. By about 9 kyr the particle falls into the sun due to rapid decrease in $q$ due to Lidov-Kozai like mechanism and eventually reaches near-ecliptic inclination $i \sim 180^{\circ}$ close to the timeframe of collision with the sun. The general behaviour evident in Figure 3 of GR-included and Newtonian-only dynamical evolution being very similar is confirmed by integration of clones (see later, where the small but significant difference will also be discussed). This trend of general dynamical behaviour in $e$ and $i$ being nearly identical between Newtonian-only and GR-included cases holds true for other bodies discussed in this work.

Figure 4 shows the future orbital evolution of ARI. Kozai like oscillations are apparent and the same pattern applies to SDA (not shown here).

Among the highest $i$ set from the 244 objects mentioned at the start of this section are the lowest perihelion distance asteroid 322P (Figure 5), designated a comet but whose characteristics such as composition, density and lack of activity point to asteroidal origin (Knight et al. 2016), and high $i$ asteroids $2008 \mathrm{KP}$ (Figure 6), 385402, 333889 and 2010 KY127. All these undergo Kozai like oscillations and GR precession at the same time. However we are unable to find any inclination flips or sun colliding phase in these bodies in the near future other than the unique example of $96 \mathrm{P}$. Hence 96P stands out in terms of its dynamical behaviour compared to all other bodies discussed in this section.

To confirm our conclusions about $96 \mathrm{P}$, we made ten clones by varying $a$ with $\pm \Delta a$ in equal steps where

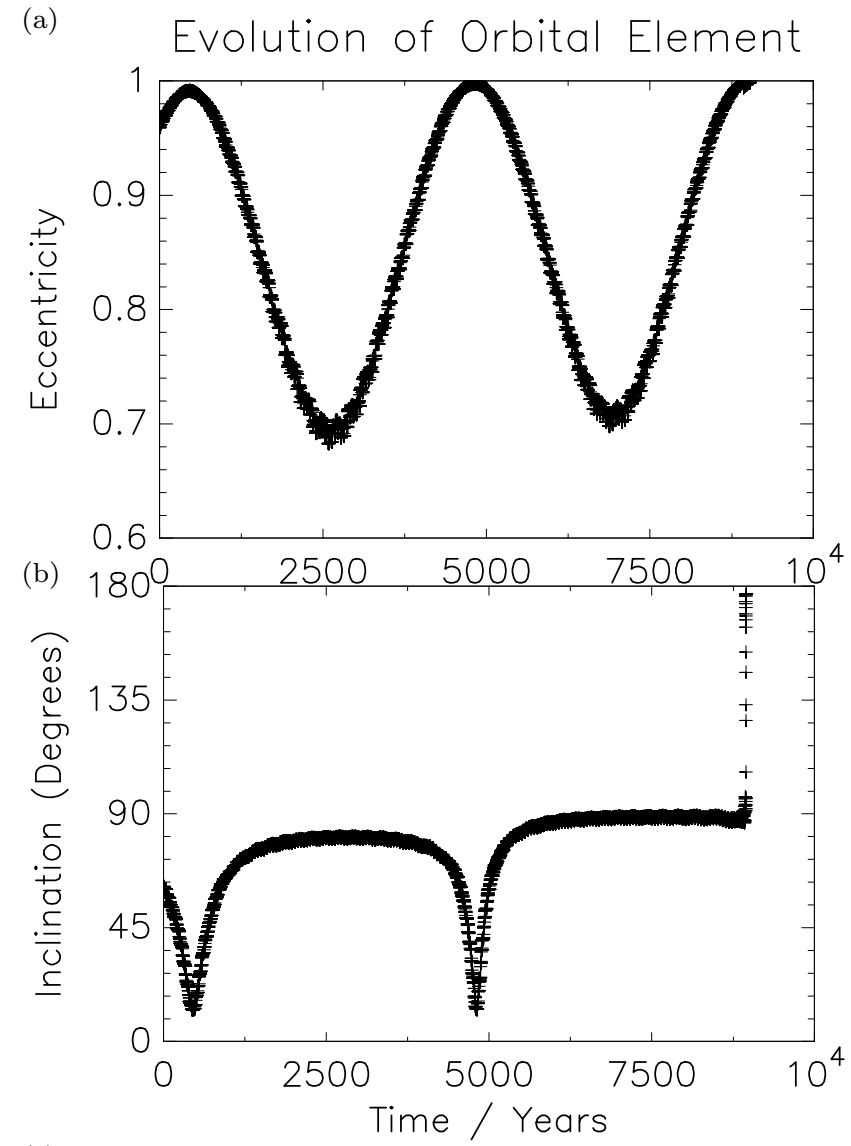

(c)

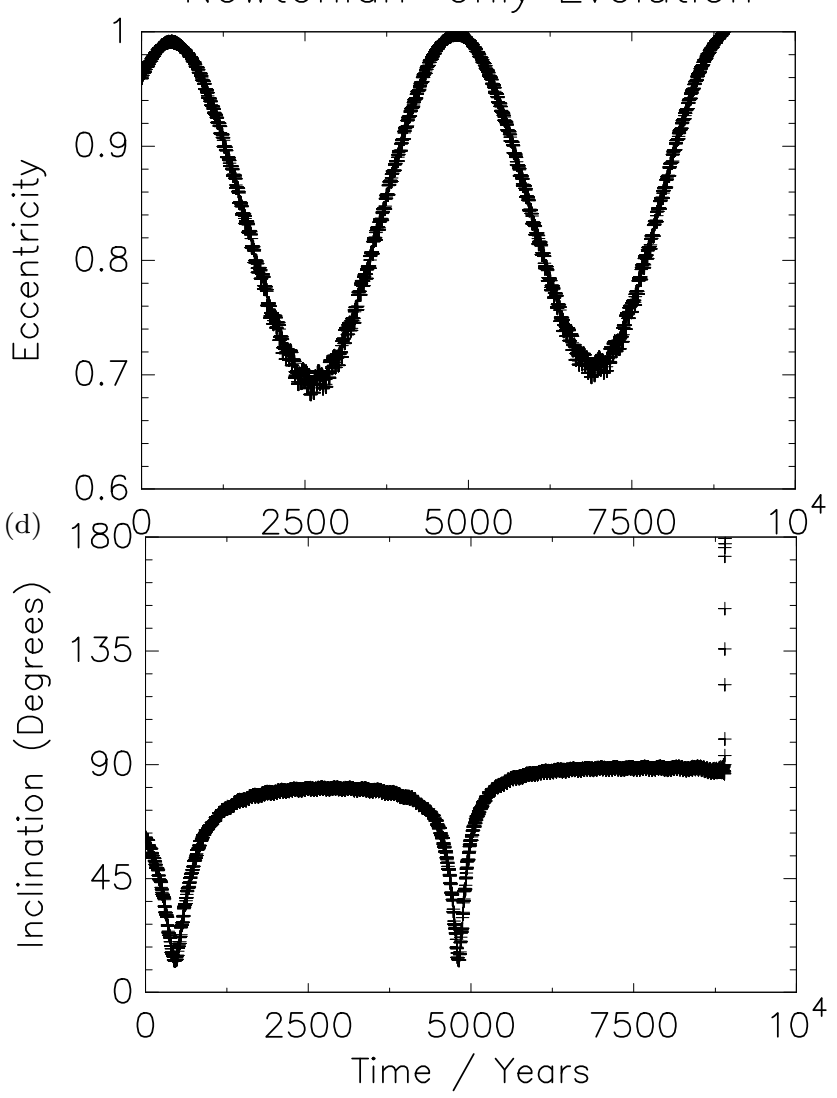

Figure 3. Orbital evolution of $(\mathrm{a}, \mathrm{c})$ eccentricity $(\mathrm{b}, \mathrm{d})$ inclination of $96 \mathrm{P}$ for $9 \mathrm{kyr}$ for GR-included and Newtonian-only cases 
Table 4. GR precession in argument of pericentre $\Delta \omega$ per century. For comparison, the GR value for Mercury is 43 arc seconds/century. The orbital elements for meteoroid streams and other minor bodies are taken from IAU-Meteor Data Center and JPL-Horizons respectively. Amongst the bodies listed here, the long term orbital evolutions for 96P, Daytime Arietids and 322P are shown in Figures 34 and 5 respectively. The short term evolutions of GR precession rates for $96 \mathrm{P}$ are shown in Figures 910 and 11

\begin{tabular}{lllll}
\hline Body & $\begin{array}{l}q \\
(\mathrm{au})\end{array}$ & $\begin{array}{l}a \\
(\mathrm{au})\end{array}$ & $\begin{array}{l}\Delta \omega \\
\text { (arcseconds } \\
\text { per century) } \\
\text { (analytic) }\end{array}$ & $\begin{array}{l}\Delta \omega \\
\text { (arcseconds } \\
\text { per century) } \\
\text { (integrations) }\end{array}$ \\
\hline \hline 96P/Machholz 1 & 0.124 & 3.035 & 3.0 & 3.7 \\
322P/SOHO & 0.054 & 2.516 & 9.0 & 9.6 \\
1566 Icarus & 0.187 & 1.078 & 10.0 & 10.6 \\
3200 Phaethon & 0.140 & 1.271 & 10.1 & 10.7 \\
Geminids & 0.2 & 1.3 & 9.3 & 9.9 \\
Daytime Arietids & 0.08 & 2.7 & 5.7 & 6.5 \\
S. Delta Aquariids & 0.07 & 0.98 & 6.7 & 7.3 \\
\hline
\end{tabular}

(a)

\section{Evolution of Orbital Element}
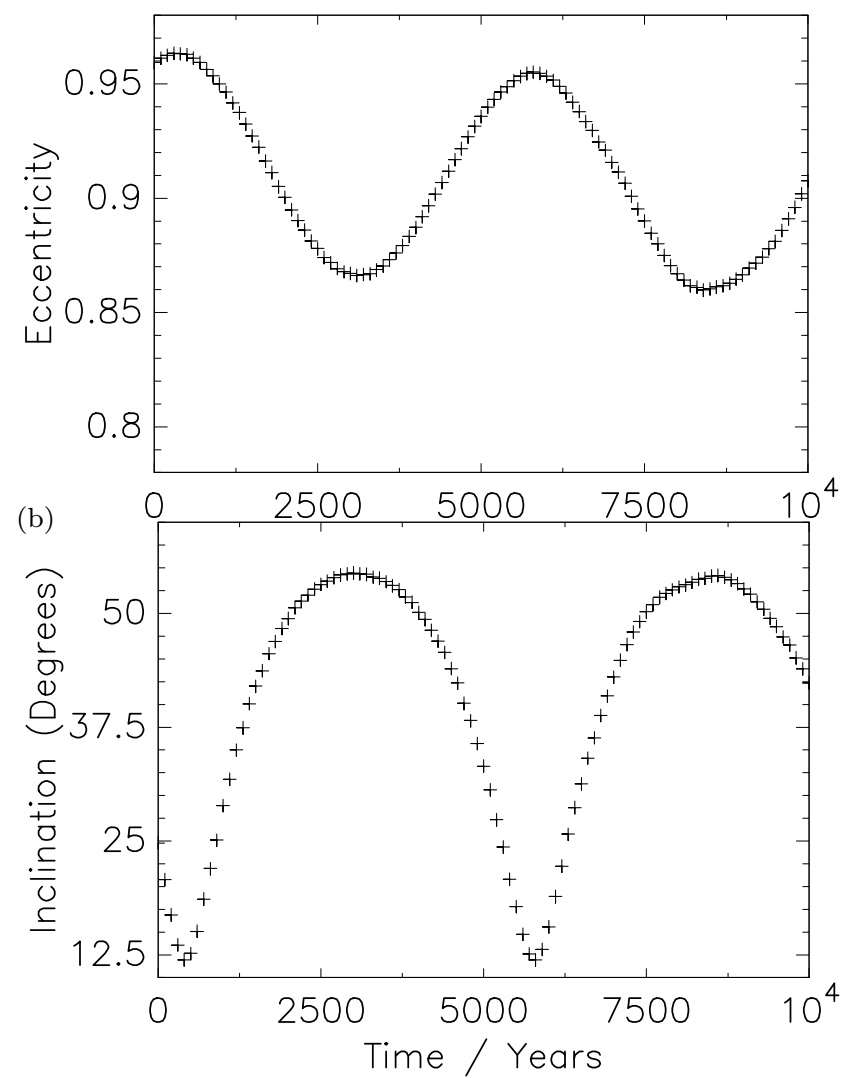

Figure 4. Evolution in $e$ and $i$ of Daytime Arietids (ARI) 10 kyr into future. Initial conditions are given in Table 1 which were taken from IAU-MDC referring to observations reported in Jenniskens et al. 2016. (a)

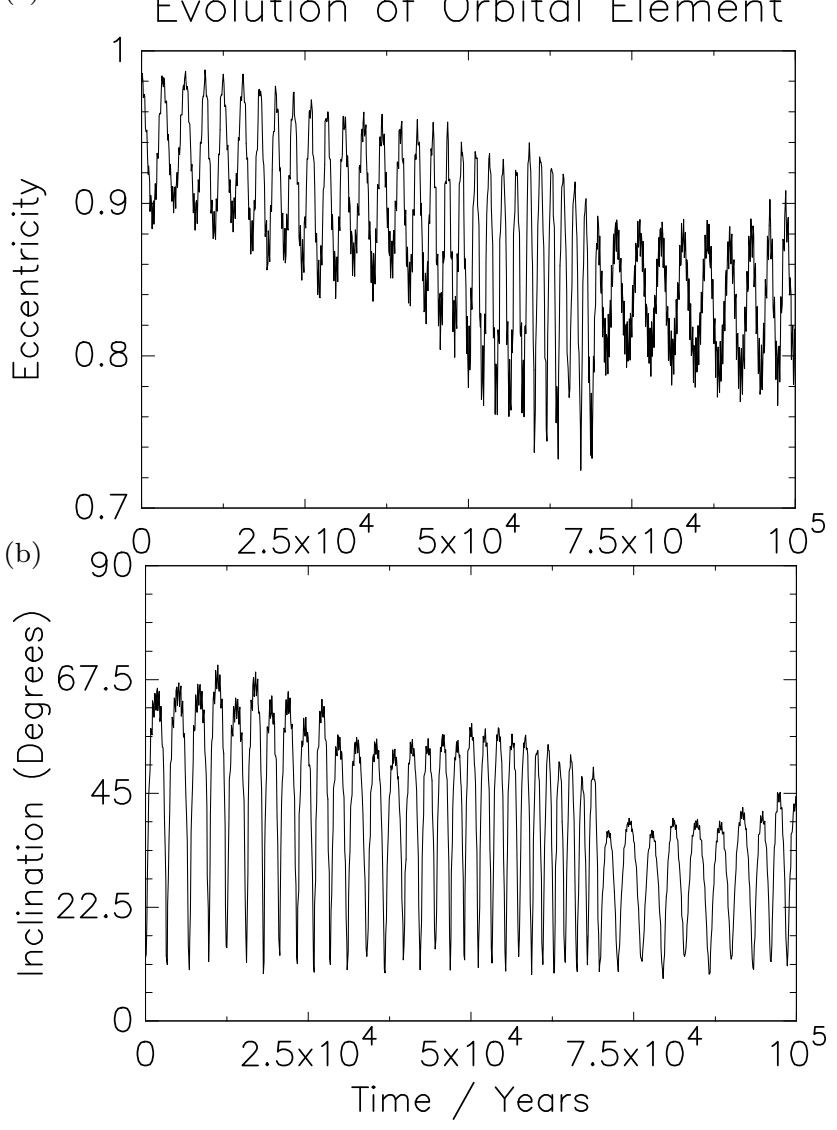

Figure 5. Orbital evolution of (a) eccentricity (b) inclination in lowest perihelion distance asteroid 322P.

$a=3.03393972$ and $\Delta a=0.0001$ au. Both Newtonian-only as well as GR-included n-body numerical tests were conducted to check how both sets of bodies dynamically evolve forward in time.

The general dynamical behaviour of any $96 \mathrm{P}$ clone is practically identical in the GR-included and Newtonian-only cases (Figure 3), looking different only for the final sungrazing and sun colliding phase (about $100 \mathrm{yr}$ for $96 \mathrm{P}$ which is shown in Figure 7). The rate of change of $e$ and $i$ is different towards the end of the bodies' evolution in the Newtonianonly and GR-included cases, with $e$ reaching 1 later (typically 5-30 yr for different clones), and $i$ reaching $180^{\circ}$ similarly later, in the GR case.

The dynamical behaviour in forward integrations stays similar for both models (which agrees with de la Fuente Marcos et al. 2015) in terms of inclination flips and sungrazing plus sun colliding phases due to Lidov-Kozai like oscillations occurring in both sets of integrations. We find that all the ten $96 \mathrm{P}$ clones survive longer $(\sim 5-30$ yr typically; shown in Figure 8) before colliding with the sun in the GR-included integrations in comparison to the ten $96 \mathrm{P}$ clones in Newtonian-only integrations. Figure 8 shows that the ten clones survive similar timescales (varying by some hundred years) before falling into the sun. But at the same time, the difference in survival times between Newtonianonly and GR-included cases are consistent for every clone.

De la Fuente Marcos et al. (2015) note that retrograde 
(a)

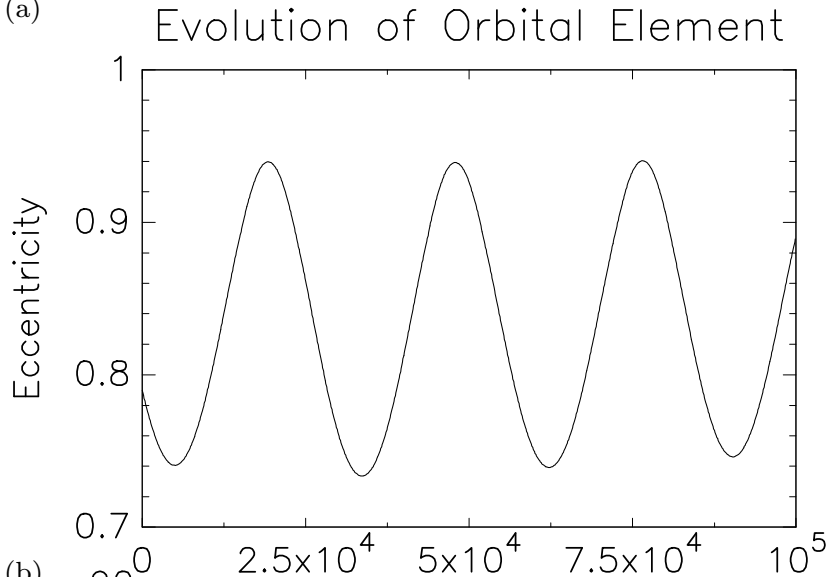

(b)

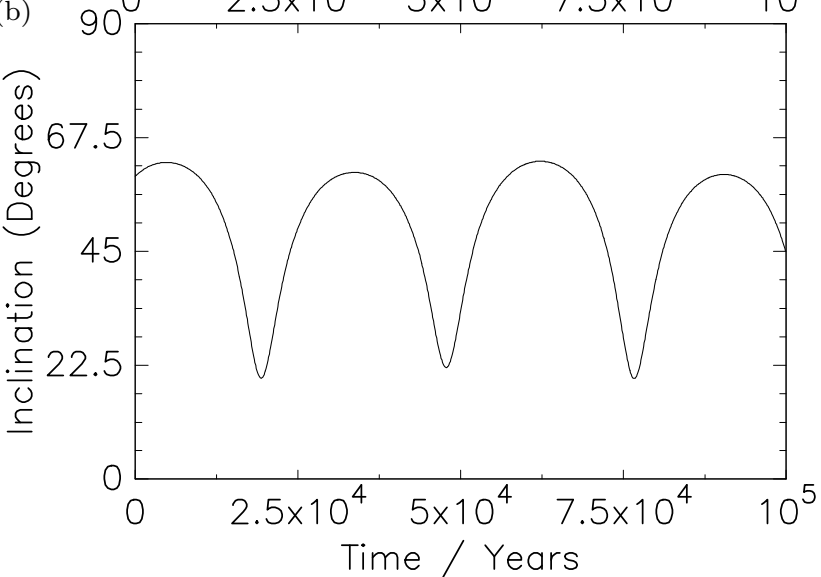

(a)

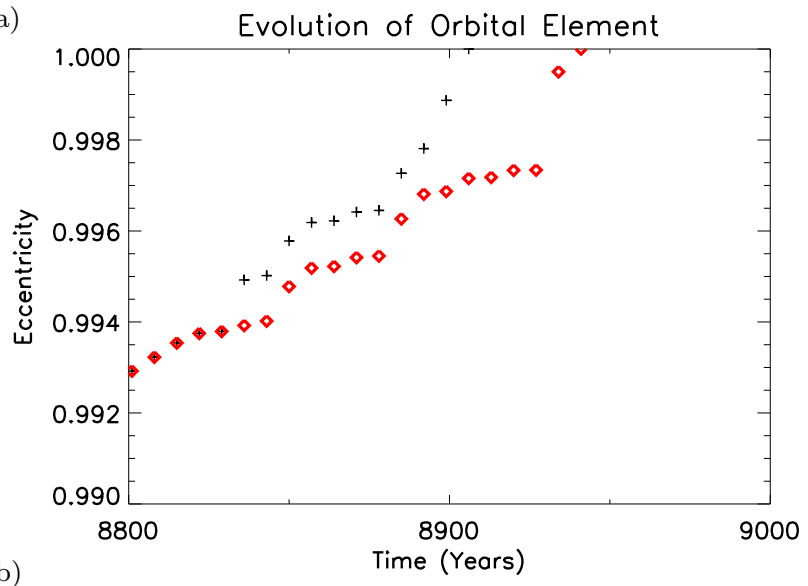

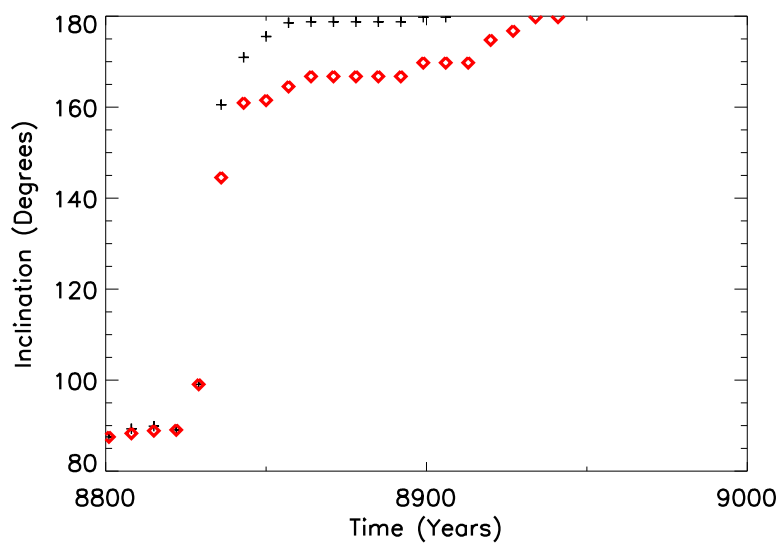

Figure 6. Evolution in $e$ and $i$ of high $i$ asteroid 2008 KP.

phases in 96P are longer in GR-included simulations compared to Newtonian-only simulations. In our simulations, the retrograde phase happens only in the final $\sim 100$ yr of $96 \mathrm{P}$ (during the same Lidov-Kozai like cycle for all clones) and we do not see any notable change in its duration between Newtonian-only and GR-included integrations.

Our integrations of $96 \mathrm{P}$ clones give an indication to the likely timeframes when possible inclination flips and sun colliding episodes could occur in future. We find that the timeframes are different from previous studies (Gonczi et al. 1992, Levison \& Dones 2014, de la Fuente Marcos et al. 2015) focused on 96P. This could be due to the relatively strong (compared to strength of close approaches with other planets like Venus, Earth, Mars and Jupiter, which we see in our integrations) close encounters with Mercury during the initial phase of our integration. The different initial conditions and orbital uncertainties have led to differences in results in previous works as well; this point has been mentioned in de la Fuente Marcos et al. (2015). Hence it is evident that exact inclination flip and sun colliding timings depend on the exact initial conditions, the type of algorithm/integrator and number of gravitational perturbers (which is different in the case of de la Fuente Marcos et al. 2015 compared to previous works) considered in the integrations. In our work, initial conditions for 96P were taken from JPL-Horizons for epoch JD 2456541.5 (which is same as in de la Fuente Marcos et al. 2015) but the particles were integrated including the gravitational effects of eight planets only (whereas de la Fuente

Figure 7. Difference in dynamical behaviour of (a) eccentricity and (b) inclination between Newtonian-only (black cross) and GR-included (red diamonds) cases, for final $\sim 100 \mathrm{yr}$ of $96 \mathrm{P}$ evolution. The particle falls into the sun earlier in the Newtonian cases compared to the GR cases.

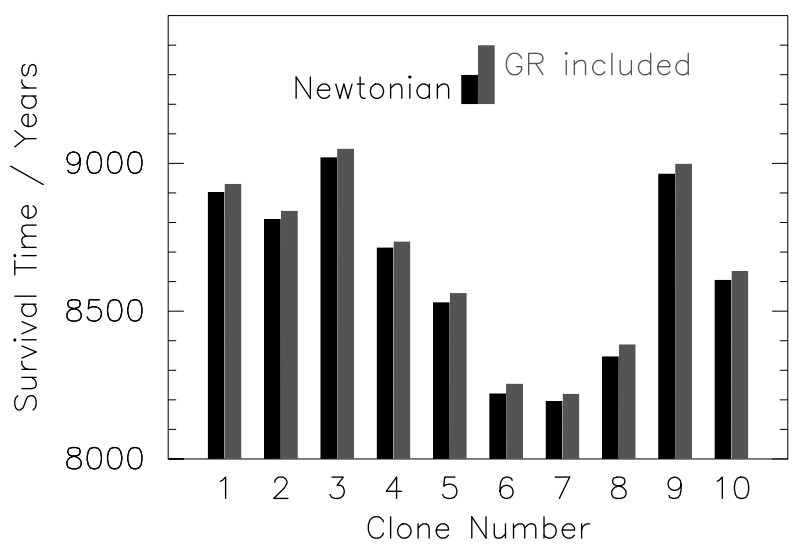

Figure 8. Survival times of both sets of Newtonian-only and GRincluded cases for all the 10 clones of 96P. The GR-included cases survive some extra years longer than Newtonian-only cases for all the clones in our integration. 
Marcos et al. 2015 included the effects of dwarf planet PlutoCharon system and the 10 most massive main belt asteroids; their initial conditions were taken in the barycentric frame from JPL-Horizons for JD 2457000.5 and used a Hermite integrator for their calculations). In our work, initial conditions for eight planets were taken in the heliocentric frame for JD 2451000.5 and we employed Mixed-Variable Symplectic or RADAU algorithms. Hence the difference in results is not surprising.

Having said this, it should be noted that irrespective of the small changes in initial conditions, all these clones undergo inclination flip and sungrazing plus sun colliding phases at some point in time (different clones showing change in times of the order of few hundreds of years typically; Figure 8), while staying within the orbital phase space where GR effects are measurable. This is the central point relevant to this work.

Because of sungrazing behaviour during the final phase of 96P's evolution, GR precession per revolution drastically increases in comparison to the initial phase of its evolution. Figures 9] and 10 show the GR precession per century and per revolution respectively; $P$ stays approximately constant. Figure 11] shows the change in GR precession for each revolution $(\sim 5 \mathrm{yr})$ in the final 100 years. These Figures are for clone 1 in Figure 8

In Figure 10] there are three intervals where GR precession rate peaks to a maximum. During 410-494 yr, GR precession $\sim 0.7$ arc seconds per revolution $(\sim 7$ times that of Mercury's GR precession). During 4650-4978 yr, GR precession stays $\geq 1.0 \mathrm{arcsec} / \mathrm{rev}(\sim 10 \times$ Mercury) with peak value $>3 \mathrm{arcsec} / \mathrm{rev}$. During 8821-8926 yr, GR precession again stays $\geq 1.0$ arcsec/rev, peak value reaching about 6 $\mathrm{arcsec} / \mathrm{rev}(\sim 60 \times$ Mercury). These extreme points in GR precession rate are due to direct effects of Lidov-Kozai like mechanism.

Our simulations show that the combination of LidovKozai like oscillations and GR precession lead to these striking sudden changes in the rate of GR precession at different points in time. In contrast with $96 \mathrm{P}$, the change in GR precession rate of Mercury itself (see Figure 12) remains almost constant throughout the same period. These are two extreme situations in the solar system in terms of this behaviour related to change in GR precession rates.

We find that Lidov-Kozai like oscillations, evolution of Keplerian orbital elements and the general dynamical behaviour remain the same in Newtonian and GR models for the bodies discussed in this work. However we were particularly interested in the rate of change of GR precession for individual bodies due to rapid changes in perihelion distances induced by Kozai oscillations and this particular pattern (due to combination of GR and Kozai) is the highlight of this study.

When Lidov-Kozai like oscillations and GR precession co-exist for the same particle, the secular decrease in $q$ caused by the former can lead to sungrazing and sun colliding phases which in turn can lead to drastically higher GR precession than at the starting time, during the final phase of these orbits (see Figures 9, 10 and 11) before they fall into the sun. This phenomenon becomes even more dynamically interesting when inclination flips occur during the final sungrazing or sun colliding phase for a body. Such a unique example in the solar system is provided by comet

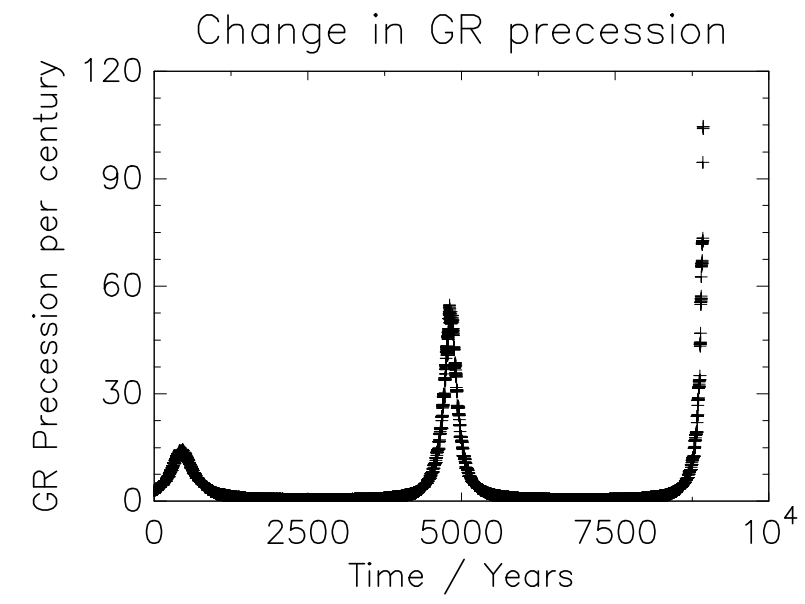

Figure 9. Change in GR precession (in arc seconds) per century for 96P/Machholz 1 for 9 kyr from present. For comparison, GR precession of Mercury is 43 arc seconds per century.

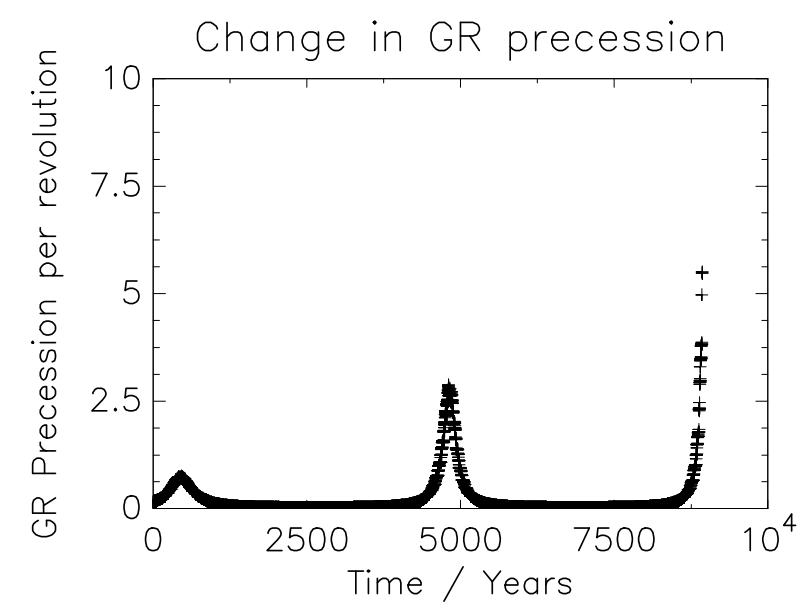

Figure 10. Change in GR precession (in arc seconds) per revolution for $96 \mathrm{P} /$ Machholz 1 for $9 \mathrm{kyr}$ from present. Drastic changes in GR precession rate occur during some intervals. For comparison, GR precession of Mercury is $0.104 \mathrm{arcsec} / \mathrm{rev}$.

96P. For the $96 \mathrm{P}$ clone having the highest contrast in GR precession rates during its evolution (see Figures 9] and 10), Table 5 lists data for the first and last $100 \mathrm{yr}$. For other timeframes in between, GR precession rates also increase and decrease rapidly, whereas for bodies like Mercury (or other terrestrial planets) GR precession per revolution stays roughly constant (Figure 12) for a long time. During the final sungrazing and sun colliding phase the GR precession for the final $100 \mathrm{yr}$ in $96 \mathrm{P}$ exceeds that of Mercury's GR precession per century. Moreover although GR precession increases substantially from 96P's initial to final sungrazing phase, conversely the Newtonian precession substantially reduces between these two phases (Table 5). Respective values for Mercury are given in the Table 5 caption for comparison.

The disparity between analytical and numerical values of GR precession during the final sungrazing phase in Table 5 is because of the rapid change (in $100 \mathrm{yr}$ ) in the body's orbit which reduces $q$ rapidly and hence is not taken into account when computing values analytically using Equation 1. On 


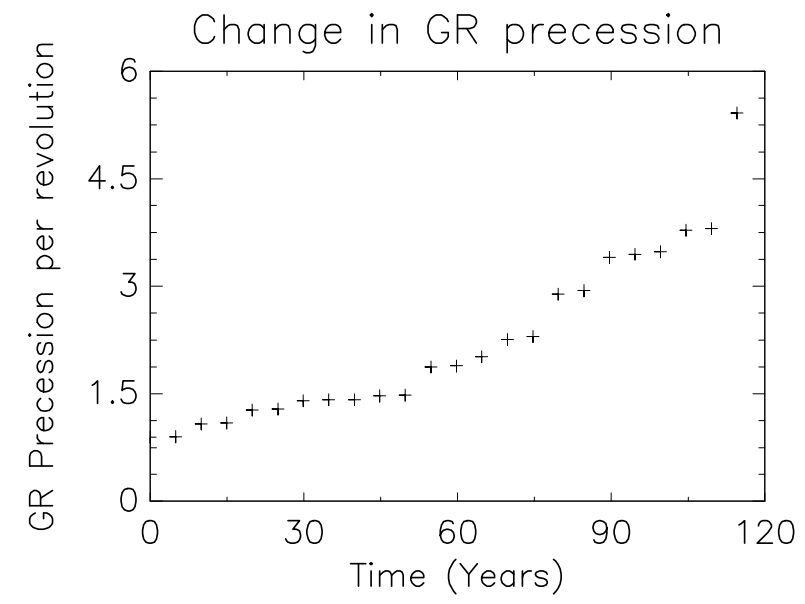

Figure 11. Change in GR precession (arcsec/rev) for 96P/Machholz 1 for about final 120 yr of same clone shown in Figure 8. There is a steady increase in GR precession rate during the final phase of a sungrazing and sun colliding orbit.

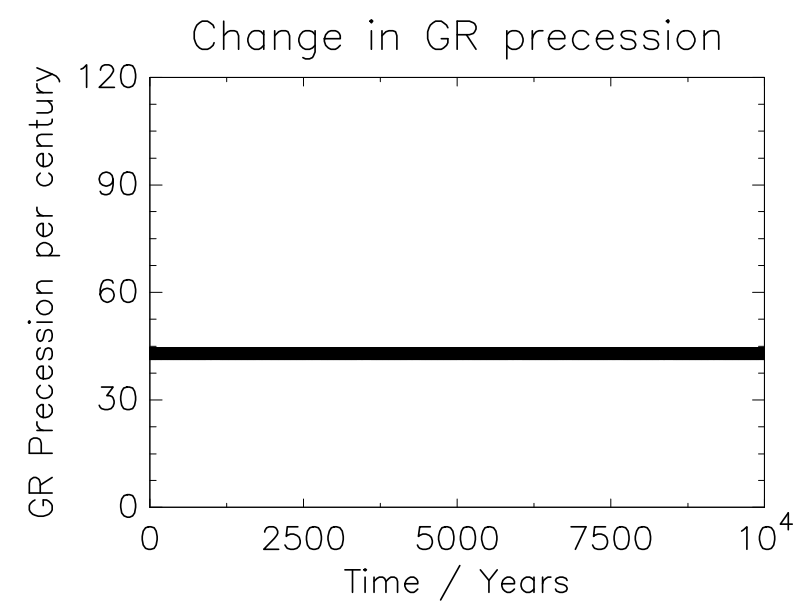

Figure 12. Planet Mercury's GR precession (arcsec/century) for 10 kyr from present.

the other hand, in numerical integrations the rapid changes in $q$ values at different time steps are correctly accounted for while calculating GR precession and hence this value is more reliable in the final sungrazing phase. Although the magnitude of Newtonian precession always exceeds GR precession at any instant of time, the Newtonian and GR precession display opposite trends during the sungrazing phase (Table 5). This behaviour is typical for all the $96 \mathrm{P}$ clones in our simulations.

Although some areas of the solar system can be extremely chaotic in general, the test particles evolving in the gravitational models we present here appear to follow a reasonably predictable pattern, that is, the evolution of orbital elements into the future is predictable to a good degree as a function of initial epoch and initial orbital elements. This is supported by the different evolution of clones with initial separation $\Delta a=0.0001$ au (survival times in Figure 8 varying up to several hundred yr), whereas with smaller $\Delta a=10^{-6}$ au (not plotted here) then the clones have practically identical dynamical evolution.
Table 5. Change in GR and Newtonian precession in argument of pericentre per century $\left(\Delta \omega / \Delta t=\omega_{t_{2}} / t_{2}-\omega_{t_{1}} / t_{1}\right)$ for a typical clone of $96 \mathrm{P}$ during initial and final hundred years of its evolution. For comparison, the GR and Newtonian precession values for Mercury are 43 and 5557 arc seconds/century (page 199, Weinberg 1972). Initial orbital elements are from JPL-Horizons and final elements from two independent numerical integrations (Newtonian-only and GR-included) performed using MERCURY package to compute the differences in $\omega$ which gives the numerical GR and Newtonian precession. $a \sim 3.034$ au during both initial and final phases of dynamical evolution discussed here.

\begin{tabular}{lllll}
\hline Body & $q$ & $\begin{array}{l}\Delta \omega / \Delta t \\
\text { (arcsec/ } \\
\text { century) } \\
\text { (analytic) } \\
\text { (GR) }\end{array}$ & $\begin{array}{l}\Delta \omega / \Delta t \\
\text { (arcseconds } \\
\text { per century) } \\
\text { (integrations) } \\
\text { (GR) }\end{array}$ & $\begin{array}{l}\Delta \omega / \Delta t \\
\text { (arcseconds } \\
\text { per century) } \\
\text { (integrations) } \\
\text { (Newtonian) }\end{array}$ \\
\hline \hline $\begin{array}{l}\text { 96P/Machholz 1 } \\
\text { (for first 100 yr } \\
\text { in the simulation) } \\
\begin{array}{l}\text { 96P/Machholz 1 } \\
\text { (for last 100 yr } \\
\text { in the simulation) }\end{array}\end{array}$ & 0.124 & 3.0 & 3.6 & 3533.0 \\
\hline
\end{tabular}

\section{CONCLUSION AND FUTURE WORK}

We have shown that there are bodies in the solar system in which both GR precession and Lidov-Kozai like mechanism can co-exist and be comparable in their effects and for which these complementary effects can be measured and identified using analytical and numerical techniques. Thus there is a continuum of bodies encompassing, firstly GR precession dominant, secondly GR precession plus Lidov-Kozai like mechanism co-existing and finally Lidov-Kozai like mechanism dominant states which are all permissible in nature. A real solar system body in this intermediate state is identified using compiled observational records from IAU-MPC, Cometary Catalogue, IAU-MDC and performing analytical plus numerical tests on them. This intermediate state brings up the interesting possibility of drastic changes in GR precession rates (at some points peaking to about 60 times that of Mercury's GR precession) during orbital evolution due to sungrazing and sun colliding phases induced by the LidovKozai like mechanism, thus combining both these important effects in a unique and dynamically interesting way. Comet 96P/Machholz 1 stands out as the only real body identified (from our simulations) to be exhibiting these interesting traits, as well as inclination flips, in the near future.

This study's purpose was to focus on the real small bodies in the solar system (including the planetary perturbations during the present epoch) exhibiting these two dynamical phenomena at the same time. For future work, it would be instructive to do a detailed abstract study, with only Jupiter so as to induce the Kozai mechanism (in its pure form) and exclude perturbations from other planets, mapping the entire Keplerian elements phase space to find the boundaries between three regions namely, GR precession dominant regime, GR precession plus Kozai mechanism co-existing regime and Kozai mechanism dominant regime. One could then compare the stability and chaotic levels 
between these three regions. This would enable us to understand the various patterns in change of GR precession rates and the maximum rates of GR precession possible in a short time for bodies in our solar system due to sungrazing phases driven by strong Lidov-Kozai like oscillations. Independently, a study of this nature could tell us the exact phase spaces which can contribute to possible sudden peaks in rates of GR precession due to Kozai like oscillations in the context of stability of artificial satellite orbits (Rosengren et al. 2015) so that precise measurements can be made for the confirmation of this combined phenomenon in future long term satellites depending on the phase space traversed.

\section{ACKNOWLEDGMENTS}

The authors thank Smadar Naoz for numerous valuable suggestions and improvements. Sekhar and Werner acknowledge the Crater Clock project (235058/F20) based at Centre for Earth Evolution and Dynamics (through the Centres of Excellence scheme project number 223272 (CEED) funded by the Research Council of Norway) and USIT UNINETT Sigma2 computational resource allocation through the Stallo cluster with project accounts Notur NN9010K, NN9283K, NorStore NS9010K and NS9029K. Research at Armagh Observatory and Planetarium is funded by the Department for Communities for N. Ireland. Vaubaillon thanks the CINES supercomputing facility of France. Li acknowledges Matt Payne for developing and providing the GR sub-routine for MERCURY.

\section{REFERENCES}

Abedin A., Wiegert P., Pokorný P., Brown P., 2017, Icarus, 281, 417.

Asher D. J., Steel D. I., 1996, MNRAS, 280, 1201.

Babadzhanov P. B., Obrubov Yu. V., 1987, in Ceplecha Z., Pecina P., eds, Proc. Vol. 2, 10th European Regional Astronomy Meeting of the IAU, Interplanetary Matter. Astron. Inst. Czechoslovak Academy of Sciences, Ondřejov, p. 141

Babadzhanov P. B., Obrubov Yu. V., 1992a, Celest. Mech. Dyn. Astron., 54, 111.

Babadzhanov P. B., Obrubov Yu. V., 1992b, in Harris A. W., Bowell E., eds, Asteroids Comets Meteors 1991, Lunar and Planetary Inst., Houston, p. 27

Babadzhanov P. B., Obrubov Yu. V., 1992c, Solar System Research, 26, 288.

Bailey M.E., Chambers J.E., Hahn G., 1992, A\&A, 257, 315.

Benitez F., Gallardo T., 2008, Celest. Mech. Dyn. Astron., 101, 289.

Boué G., Laskar J., Farago F., 2012, A\&A, 548, A43.

Brumberg V., 1991, Essential Relativistic Celestial Mechanics. Adam Hilger, London

Chambers J. E. 1999, MNRAS, 304, 793.

de la Fuente Marcos C., de la Fuente Marcos R., Aarseth S. J., 2015, MNRAS, 446, 1867.

Einstein A. 1915, Preussische Akademie der Wissenschaften, Sitzungsberichte, 831.

Everhart E., 1985, in Carusi A., Valsecchi G. B., eds, Astrophysics and Space Science Library, Vol. 115 (Proc. IAU Colloq. 83), Dynamics of Comets: Their Origin and Evolution. Reidel, Dordrecht, p. 185

Fox K., Williams I.P., Hughes D.W. 1982, MNRAS, 199, 313.

Galushina T. Yu., Ryabova G. O., Skripnichenko P. V., 2015, Planet. Space Sci., 118, 296.

Giorgini J. D. et al., 1996, BAAS, 28, 1158

Gonczi R., Rickman H., Froeschlé C., 1992, MNRAS, 254, 627.

Granvik M., Vaubaillon J., Jedicke R., 2012, Icarus, 218, 262.
Iorio L. 2005, A\&A, 433, 385.

Jenniskens P. et al., 2016, Icarus, 266, 331.

Kerr R. A., 1992, Science, 258, 27.

Knight M. M., Fitzsimmons A., Kelley M. S. P., Snodgrass C., 2016, ApJ, 823, L6.

Kozai Y. 1962, AJ, 67, 591.

Kreutz H. 1888, Astronomische Nachrichten, 119, 381.

Levison H. F., Dones L., 2014, in Spohn T., Breuer D., Johnson T. V., eds, Encyclopedia of the Solar System, 3rd edn. Elsevier, Amsterdam, p. 705

Li G., Naoz S., Kocsis B., Loeb A., 2014a, ApJ, 785, 116.

Li G., Naoz S., Holman M., Loeb A., 2014b, ApJ, 791, 86.

Li G., Naoz S., Kocsis B., Loeb A., 2015, MNRAS, 451, 1341.

Lidov M. L., 1962, Planet. Space. Sci., 9, 719.

Lithwick Y., Naoz S., 2011, ApJ, 742, 94.

Lithwick Y., Wu, Y., 2014, Proc. Natl. Acad. Sci., 111, 12610.

Manley S. P., Migliorini F., Bailey M. E. 1998, A\&AS, 133, 437.

Marsden B. G., Williams G. V., 2008, Catalogue of Cometary Orbits, 17th ed. Minor Planet Center/Central Bureau for Astronomical Telegrams, Cambridge, MA.

Morbidelli A., 2011, Modern Celestial Mechanics: Aspects of Solar System Dynamics. Taylor \& Francis/Cambridge Scientific Publishers.

Naoz S., Farr W. M., Lithwick Y., Rasio F. A., Teyssandier J., 2011, Nature, 473, 187.

Naoz S., Kocsis B., Loeb A., Yunes N., 2013, ApJ, 773, 187.

Naoz S. 2016, ARA\&A, 54, 441.

Ohtsuka K., Nakano S., Yoshikawa M., 2003, PASJ, 55, 321.

Quinn T. R., Tremaine S., Duncan M., 1991, AJ, 101, 2287.

Rosengren A. J., Alessi E. M., Rossi A., Valsecchi G. B., 2015, MNRAS, 449, 3522 .

Sekanina Z., Chodas P. W., 2005, ApJS, 161, 551.

Sekhar A., 2013, WGN (J. Int. Meteor Organization), 41, 179.

Shahid-Saless B., Yeomans D.K., 1994, AJ, 107, 1885.

Sitarski G., 1992, AJ, 104, 1226.

Vaubaillon J., Lamy P., Jorda L., 2006, MNRAS, 370, 1841.

Weinberg S. 1972, Gravitation and Cosmology: Principles and Applications of the General Theory of Relativity. Wiley, New York

Werner S. C., Ivanov B. A., 2015, in Schubert G., editor-in-chief, Treatise on Geophysics, 2nd edition, Vol. 10. Elsevier, Oxford, p. 327

Wisdom J., Holman M., Touma J., 1996, Fields Inst. Commun., 10, 217. 